\title{
Sub-deg-per-hour Edge-Anchored Bulk Acoustic Wave Micromachined Disk Gyroscope
}

\author{
Madan Parajuli, Student Member, IEEE, Guillermo Sobreviela, Milind Pandit, Hemin Zhang, \\ and Ashwin A. Seshia, Fellow, IEEE
}

\begin{abstract}
This paper reports on the experimental characterization of a vacuum packaged bulk acoustic wave disk gyroscope with T-shape anchors fabricated on a (100) single-crystal silicon substrate. Improvements in key device performance parameters are achieved by implementing mode matching using electrostatic frequency tuning to decrease the frequency split between two near-degenerate bulk acoustic modes from $4 \mathrm{~Hz}$ to $0.4 \mathrm{~Hz}$. Gyroscopic operation is demonstrated by driving one of the secondary degenerate elliptical modes while sensing the response in the other elliptical mode, both demonstrating quality factors exceeding 1 million at an operating frequency of $\sim 976 \mathrm{kHz}$. As a result, the mechanical sensitivity of the gyroscope is improved by a factor of $\mathbf{7 . 7 6}$ times for near mode-matched operation relative to the case with no tuning of the frequency spacing between the modes. Additionally, the angle random walk and bias instability were improved from $0.042 \% \sqrt{h}$ to $0.037 \% \sqrt{h}$ and $1.65 \%$ to $0.95 \% / h$ respectively, benchmarking favorably with respect to the state-of-the-art of BAW disk gyroscopes.
\end{abstract}

Index Terms-MEMS, Gyroscopes, Bulk acoustic wave mode, Mode matching

\section{INTRODUCTION}

MEMS gyroscopes are widely used in a variety of applications including motion sensing in consumer electronics, automobiles, and robotics, due to their advantageous features such as small size, low power consumption, robustness, and low cost due to volume manufacturing considerations. Recent improvements in resolution and long-term stability are addressing suitability of MEMS gyroscopes for higher-end applications, such as pedestrian navigation, navigation systems of unmanned aerial and underwater vehicles, and motioncompensated imaging systems.

A MEMS vibratory gyroscope utilizes the interaction between two or more vibratory modes of a device in a rotating reference frame due to the Coriolis effect in order to provide a measurement of angular rate about a given axis. In recent years, extensive research has been conducted on high performance gyroscopes with different structures, such as the disk resonator gyroscope (DRG) [1], [2], bulk-acoustic wave (BAW) gyroscope [3], quad mass (QMG) [4], and birdbath gyroscope (BRG) [5], [6]. While the chosen topology can be very different, all of these different designs have leveraged a combination of attributes: structural symmetry, high $\mathrm{Q}$ factors

Manuscript received July 15, 2020; revised August 13, 2020; accepted August 17, 2020.

M. Parajuli, H. Zhang and A. A. Seshia are with The Nanoscience Centre, Department of Engineering, University of Cambridge, Cambridge CB3 OFF, U.K. email:mp912@cam.ac.uk

G. Sobreviela and M. Pandit are with Silicon Microgravity Ltd., Cambridge Innovation Park, Cambridge CB25 9PB, U.K associated with drive/sense modes of interest that are closely matched prior to tuning and optimizing other relevant features such as drive velocity amplitude and environmental immunity to shock and vibration and/or temperature, integration with low-noise readout electronics and active control of the response in the drive/sense modes.

In comparison to lower frequency flexural gyroscopes, BAW gyroscopes can demonstrate higher quality factors as the bulk modes are significantly less impacted by thermoelastic damping (TED). In addition, bulk mode devices display higher stiffnesses and resonant frequencies - often by at least 2 orders of magnitude relative to their flexural mode counterparts. This increases the shock and vibration robustness of such devices. Additionally, BAW modes are less susceptible to air damping compared to flexural gyroscopes and Q factors saturate at higher pressure levels alleviating the demands on vacuum packaging. These characteristics make BAW gyroscopes potential candidates for high-end applications.

Previous works on BAW gyroscopes have been based around a silicon micromachined disk supported by a central anchor [3], [7]. This approach relies on well-controlled sacrificial layer etching with etch holes built into the disk for front-side structural release. The addition of etch holes on the disk can degrade the quality factor of the modes of interest. Additionally, fabrication tolerances associated with etch release can vary the dimension of the central anchor and/or result in misalignments in position. This can break structural symmetry resulting in an increased frequency split and asymmetry in damping associated with the drive and sense modes. In contrast, the edge-anchored designs simplify fabrication requirements and provides robustness of process tolerances by enabling design of all critical dimensions (including the anchors) in a single-step DRIE etch. This approach can reduce the impact of process tolerances on the frequency split between drive and sense modes [8]. Edge-anchored BAW resonators have previously demonstrated high quality factors under vacuum conditions and oscillators based on high-Q resonant modes have demonstrated excellent frequency stability [9]-[12].

The anisotropic nature of (100) single crystal silicon (SCS) results in a frequency split associated with the two primary wine glass modes making them readily suitable for gyroscopic application. Although the use of an isotropic material, such as polysilicon or use of (111) SCS wafers could allow the use of primary wine glass modes for angular rate sensing [2], (100) SCS offers advantages due to its ready availability as a substrate material. Gyroscopic operations in devices made 
of (100) SCS are possible using the higher-order elliptical modes as they exhibit degeneracy [13], [14]. Fabrication tolerances [8], material anisotropy [14] outlined in previous studies, and practical considerations associated with anchoring arrangements can result in a non-zero frequency split between the degenerate modes. In such cases, electrostatic frequency tuning can be employed to achieve mode matching [8]. Due to the high mechanical stiffnesses associated with the BAW modes, it can be difficult to compensate for even small mismatches in the natural frequencies: in order to overcome this problem, understanding the alignment of the mode shapes with the peripheral electrodes is necessary as it enables a systematic approach to mode matching using electrostatic frequency tuning, details of which are presented elsewhere [15].

This paper demonstrates an improvement in sensitivity of a BAW MEMS gyroscope by implementing mode-matching. The principle of operation of the gyroscope is presented in section II. Section III describes the device and its COMSOL simulation results. The experimental setup is described in section IV. Results, verifying the advantages of mode-matching are shown in section V. Finally, conclusions are presented in section VI.

\section{PRINCIPLE OF OPERATION}

A summary of the basic working of Coriolis vibratory rate gyroscopes has been covered previously [16]-[18]. The simplest abstraction of a single-axis Coriolis vibratory rate gyroscope is the lumped element two degrees-of-freedom (2-DOF) mass-spring-dashpot model shown in Fig. 1. The simplified 2-DOF equations of motion can be expressed as:

$$
\begin{aligned}
m \ddot{x}+c_{x} \dot{x}+k_{x} x & =\tau_{x}+2 m \Omega_{z} \dot{y} \\
m \ddot{y}+c_{y} \dot{y}+k_{y} y & =\tau_{y}-2 m \Omega_{z} \dot{x}
\end{aligned}
$$

where $\tau_{x}$ and $\tau_{y}$ are the external forces in drive and sense direction, respectively; $\Omega_{z}$ is the rate of rotation applied about an axis normal to the plane of modal vibration; $m$ is the effective mass; $k_{x}$ and $k_{y}$ are stiffness in the drive and sense direction; $c_{x}$ and $c_{y}$ are damping in the drive and sense direction. The term $2 m \Omega_{z} \dot{x}$ is the rotation induced Coriolis force. In open loop sense mode operation, $\tau_{y}=0$ and $2 m \Omega_{z} \dot{y}$ is negligible since the drive mode amplitude is higher than the sense mode response. The Coriolis force term $2 m \Omega_{z} \dot{x}$ induces sense mode response proportional to the angular rotation. The mechanical sensitivity $\left(\frac{y_{0}}{\Omega_{z}}\right)$ which is the ratio of the sense mode response to angular rotation $\left(\Omega_{z}\right)$ as a function of frequency split $(\Delta f)$ between drive mode resonant frequency $\left(f_{d}\right)$ and sense mode resonant frequency $\left(f_{s}\right)$ can be expressed as:

$$
\frac{y_{0}}{\Omega_{z}}=\frac{f_{d}}{\pi\left(f_{d}+\Delta f\right)^{2}} \frac{x_{0}}{\sqrt{\left[1-\left(\frac{f_{d}}{\left(f_{d}+\Delta f\right)}\right)^{2}\right]^{2}+\left[\frac{f_{d}}{Q_{s}\left(f_{d}+\Delta f\right)}\right]^{2}}}
$$

where $x_{0}$ is the drive mode amplitude and $Q_{s}$ is the sense mode quality factor. The mechanical sensitivity can be significantly improved by reducing the frequency split to zero where the frequencies of the drive and sense modes are precisely matched. The mechanical sensitivity at mode-matched operation can be obtained by substituting $\Delta f=0$ in equation 2:

$$
\frac{y_{0}}{\Omega_{z}}=\frac{Q_{s} x_{0}}{\pi f_{d}}
$$

Mode-matched operation is a route towards higher perfor-

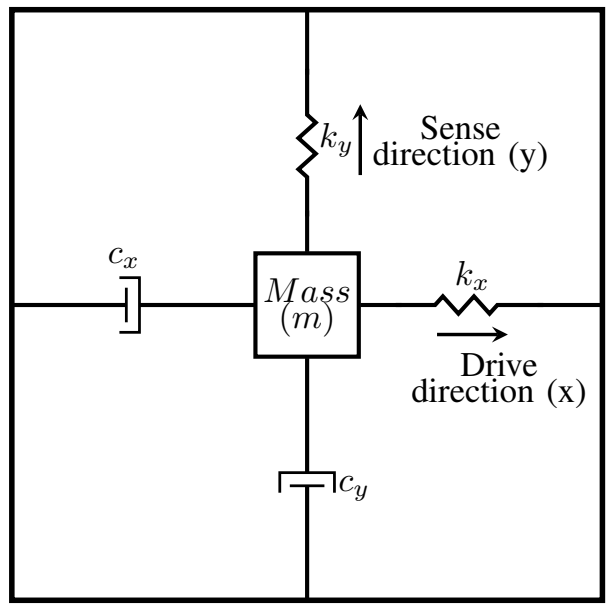

Fig. 1. A 2-DOF mass-spring-damper model for a single-axis Coriolis rate gyroscope.

mance by maximizing the mechanical sensitivity and consequently leading to the potential for low noise and high stability measurements [19], [20]. Under such conditions an approximate analytical expression for thermo-mechanical limited angular rate noise is given by [21]:

$$
\Omega_{(\text {Brownian })} \propto \frac{1}{x_{0}} \sqrt{\frac{4 k_{B} T}{\omega_{0} m_{\text {eff }} Q_{\text {effect-sense }}}}
$$

where $x_{0}$ is the drive amplitude; $\omega_{0}, m_{\text {eff }}$ and $Q_{\text {effect-sense }}$ are the resonant frequency, effective mass and effective quality factor at the sense mode, respectively; $k_{B}$ is the Boltzmann constant and $T$ is the absolute temperature. Equation 4 shows that the noise floor can be improved by increasing the quality factor and the resonant frequency.

\section{DEVICE}

The device-under-test in this study is a vacuum packaged micromachined disk gyroscope fabricated in a (100) single crystal silicon (SCS) substrate using a silicon-on-insulator (SOI) MEMS process employing wafer-level vacuum encapsulation [22]. The characterisation of resonant modes for this device has revealed several modes with quality factors exceeding a million [10], [15] and a Q-f metric exceeding $10^{13}$.

Fig. 2 shows an optical micrograph of the device. The disk has a radius of $3 \mathrm{~mm}$ and a thickness of $40 \mu \mathrm{m}$. The disk is surrounded by 12 identical electrodes, and employs edge anchoring [8]. The secondary degenerate modes illustrated in Fig. 3 (generated by the finite element analysis (COMSOL) simulation results of these modes) are used to measure angular rotation. The mode shapes and resonant frequencies of inplane vibration modes can be estimated theoretically using 


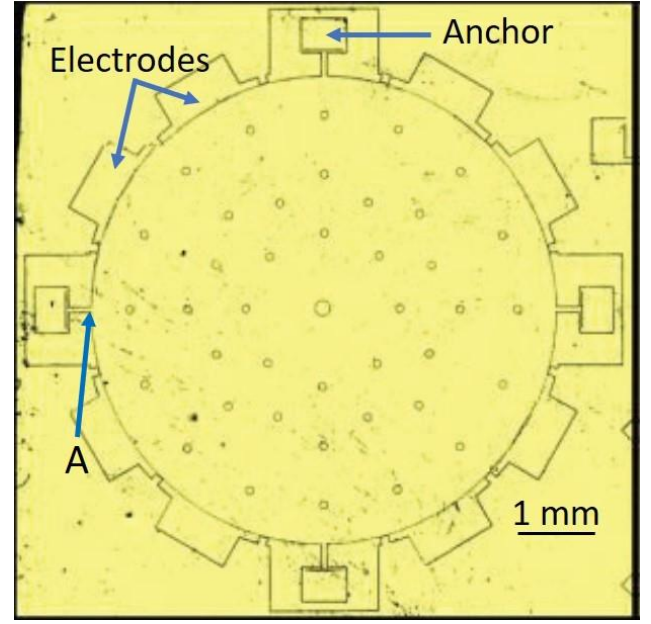

Fig. 2. Optical micrograph of the MEMS device.

two dimensional elastic theory [23]. Reference [15] presents the systematic approach to experimentally characterize these modes. The key design parameters and simulation results of the device are summarized in Table I.
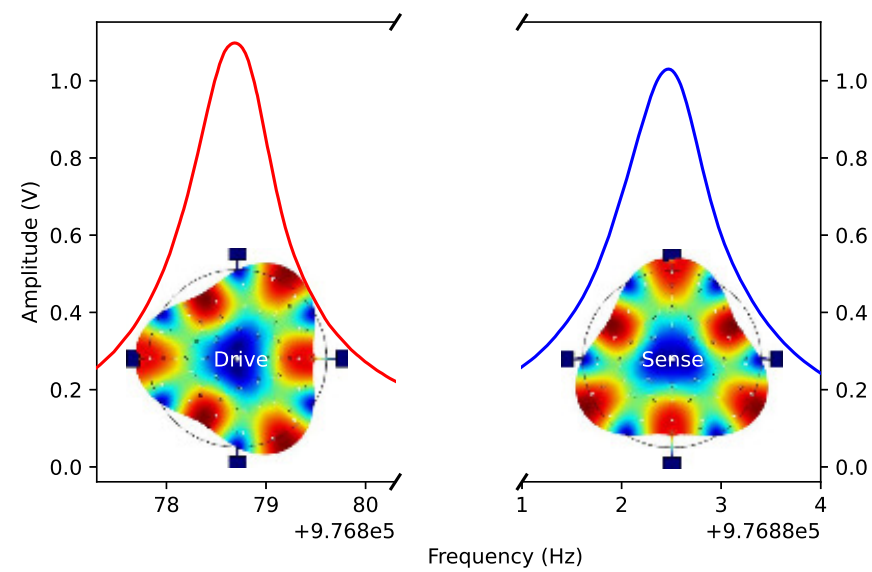

Fig. 3. Frequency response of the drive and sense mode of the gyroscope and the corresponding COMSOL simulation results.

TABLE I

SUMMARY OF DESIGN PARAMETERS OF THE DEVICE

\begin{tabular}{|c|c|c|}
\hline \multicolumn{2}{|c|}{ Design Parameters } & Value \\
\hline \multicolumn{2}{|c|}{ Disk radius } & $6 \mathrm{~mm}$ \\
\hline \multicolumn{2}{|c|}{ Device thickness } & $40 \mu \mathrm{m}$ \\
\hline \multirow{4}{*}{ T- shape suspension } & Horizontal beam width & $25 \mu \mathrm{m}$ \\
\hline & Horizontal beam length & $480 \mu \mathrm{m}$ \\
\hline & Vertical beam width & $60 \mu \mathrm{m}$ \\
\hline & Vertical beam length & $295 \mu \mathrm{m}$ \\
\hline \multicolumn{2}{|c|}{$\begin{array}{l}\text { Trigonal mode frequencies } \\
\text { (COMSOL simulation) }\end{array}$} & $\begin{array}{l}998781 \mathrm{~Hz} \\
998782 \mathrm{~Hz}\end{array}$ \\
\hline
\end{tabular}

The total Quality factor is determined by the contribution of different sources of energy loss such as thermoelastic damping (TED) [24], anchor loss [25], squeeze-film damping (SFD) [26] and other intrinsic losses [27]. Table II summarises the simulated results for the TED and anchor loss for this specific design. Fluid damping is negligible due to vacuum encapsulation which is implemented at the wafer level [22]. Anchor loss is estimated through numerical finite element simulations using a perfectly matched layer (PML) [28] approach. The estimated total quality factor matches closely with the measured results. The difference in the $\mathrm{Q}$ factor between two modes can be related with meshing limitations of the simulation software.

TABLE II

ESTIMATED QUALITY FACTOR FOR THE SECONDARY DEGENERATE MODES

\begin{tabular}{|c|c|c|}
\hline Parameter & Drive mode & Sense mode \\
\hline Thermoelastic damping, $\mathrm{Q}_{T E D}$ & 4008022 & 4004750 \\
\hline Anchor damping, $\mathrm{Q}_{\text {anchor }}$ & 2682489 & 2688565 \\
\hline Total loss, $\mathrm{Q}_{\text {total }}$ & 1606973 & 1608625 \\
\hline
\end{tabular}

Evolution of the mode shapes of interest due to added stiffness perturbation (resulting from electrostatic tuning for instance) is modelled using finite element analysis (COMSOL). Fig. 4 shows the total in-plane displacement of a point at the periphery of a disk (point A in Fig. 2) for both modes as a function of added stiffness perturbations. These simulations are employed to understand the evolution of the response with perturbations due to geometric imperfections, material anisotropy and anchoring arrangements, as well as to model the effect of stiffness variations due to electrostatic loads.

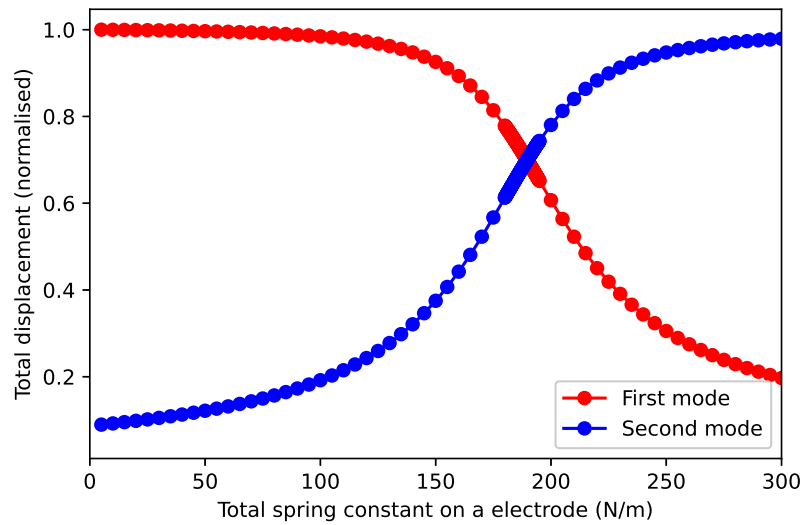

Fig. 4. The total in-plane displacement of a point at the periphery of a disk (point A in Fig. 2) as a function of added stiffness perturbation.

Initially, the device is experimentally characterized in an open-loop configuration: Fig. 3 shows the measured frequency response of the drive mode (red trace) and the sense mode (blue trace) when each mode is individually excited. The resonant frequencies for the secondary degenerate elliptical modes of this device are $976878.5 \mathrm{~Hz}$ and $976882.5 \mathrm{~Hz}$ respectively, with an initial frequency split of $4 \mathrm{~Hz}$.

Fig. 5a and 5b plot the amplitude decay of the output voltages corresponding to the drive and sense modes of the gyroscope during separate ring-down experiment. The ringdown time $(\tau)$ for the drive mode (lower frequency mode) and sense mode (higher frequency mode) are $0.43 \mathrm{~s}$ and 0.51 $\mathrm{s}$ respectively. This corresponds to a quality factor of $1.31 * 10^{6}$ 


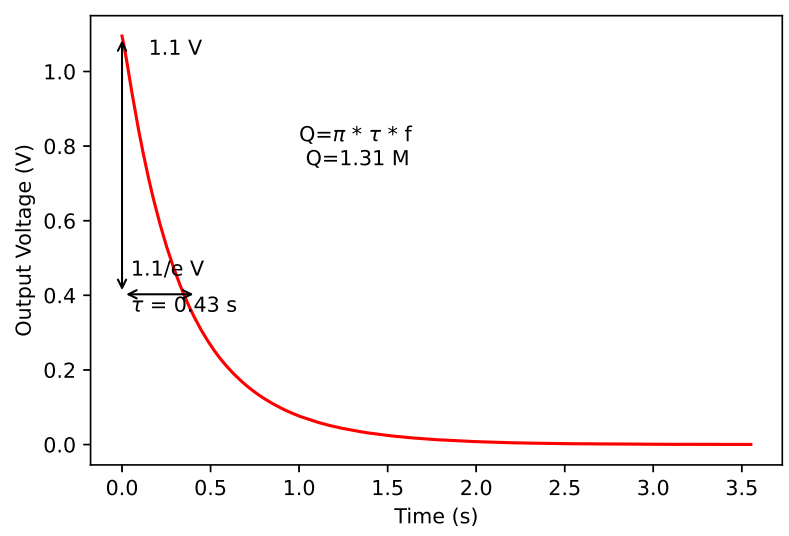

(a)

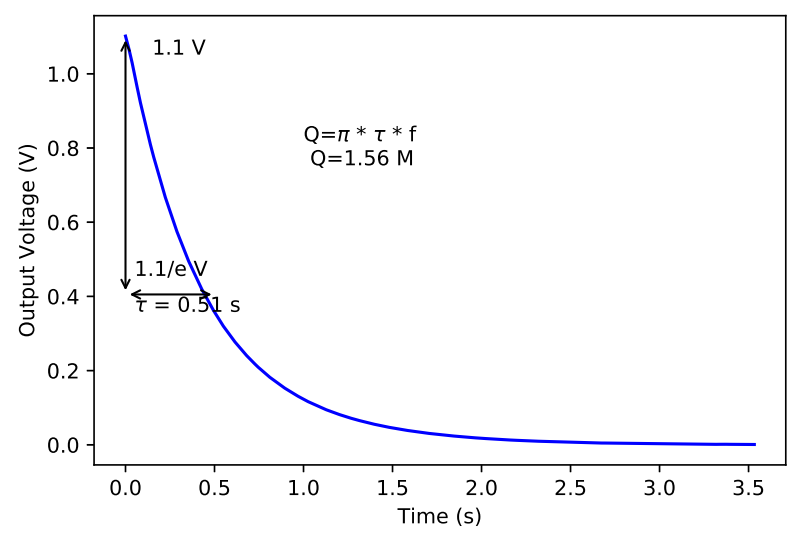

(b)

Fig. 5. Measured ring down response of (a) the drive mode and (b) the sense mode.

and $1.56 * 10^{6}$ respectively which is in reasonable agreement with the theoretical prediction outlined in Table II. A key figure of merit for gyroscopes is related to the f.Q product. For the specific device-under-test, this value is $1.5 * 10^{12}$ which benchmarks favorably with respect to the state-of-the-art [10], [29].

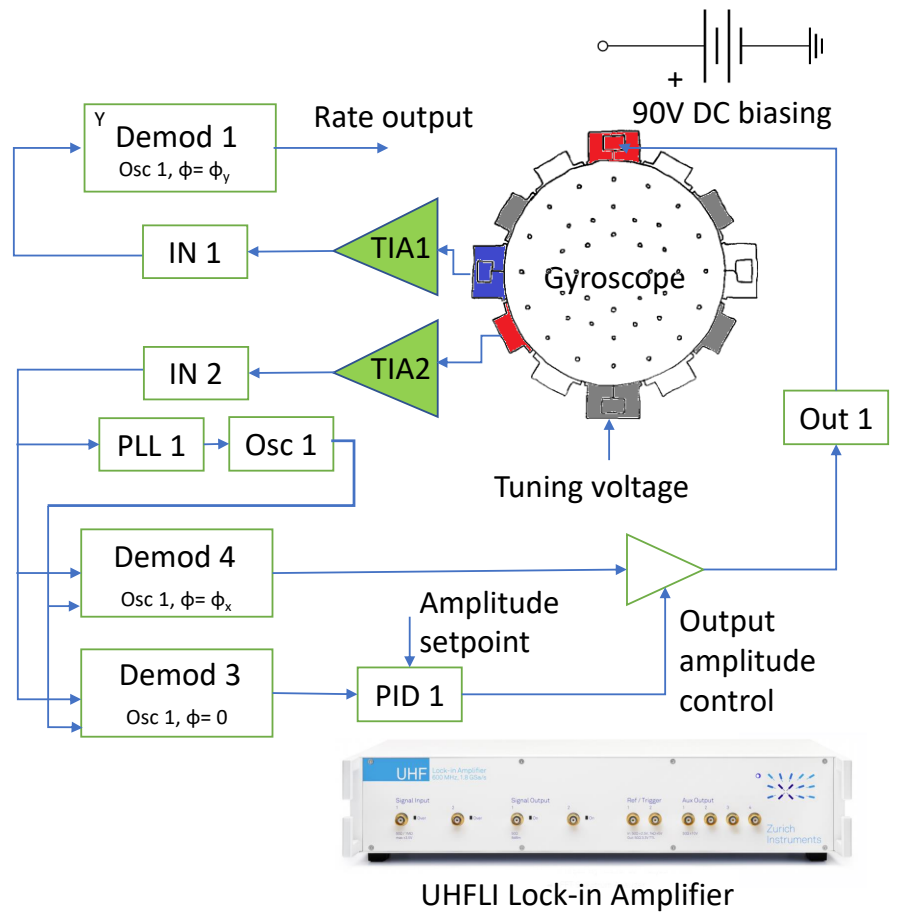

Fig. 6. Schematic of experimental setup.

\section{EXPERIMENTAL SETUP}

A schematic of the experimental setup is shown in Fig. 6. The drive electrode for the device is connected directly to the output of the UHFLI and a second electrode is employed to record the motional current corresponding to the drive mode. This motional current is amplified by a transimpedance amplifier (TIA) and then connected to the input of the UHFLI system. A bias voltage of $90 \mathrm{~V}$ is supplied to the proof mass and substrate of the resonator.

The oscillation of the drive mode is maintained at resonance by means of the phase locked loop function of UHFLI. The phaseshift $\phi_{x}$ of demodulator 4 is set to maximize the amplitude of oscillations. In addition, amplitude gain control (AGC) is implemented to keep the mechanical amplitude of the drive mode constant. The AGC implementation uses a PID controller to compare and regulate the drive mode oscillation amplitude to a given setpoint.

The sense mode is implemented in open loop. The input of demodulator 1 is connected through the TIA to the sense mode output of the gyroscope. Demodulator 1 uses oscillator 1 , which is controlled by the PLL. The demodulator phase shift $\phi_{y}$ is adjusted to ensure that the component $\mathrm{Y}$ of demodulator 1 is the Coriolis output.

\section{REsults}

Electrostatic frequency tuning is employed in the device to match the frequencies of the drive and sense modes. Initially, the resonator body is biased relative to the surrounding electrodes which are grounded resulting in an initial frequency offset due to the resulting stiffness tuning. To achieve mode matching, the resonant frequency of the first mode is increased to match the resonant frequency of the second mode. By supplying a positive tuning voltage on the electrodes aligned to the antinodes of the first mode, the corresponding electrical stiffness is reduced, increasing its resonant frequency to match that of the second mode. A tuning voltage of $33.7 \mathrm{~V}$ was applied on the 4 electrodes marked in gray in Fig. 6 to decrease the frequency split from $4.06 \mathrm{~Hz}$ to $0.4 \mathrm{~Hz}$. Fig. $7 \mathrm{a}$ and $7 \mathrm{~b}$ show the initial frequency response and the tuned frequency response of the device for comparison.

A single-axis motion simulator (Acutronic model $\mathrm{AC} 1120 \mathrm{Si}$ ) is used to test the response of the device to rotational motion. In a specific experiment, the rotation rates are varied as steps inputs with rotation rate levels successively 


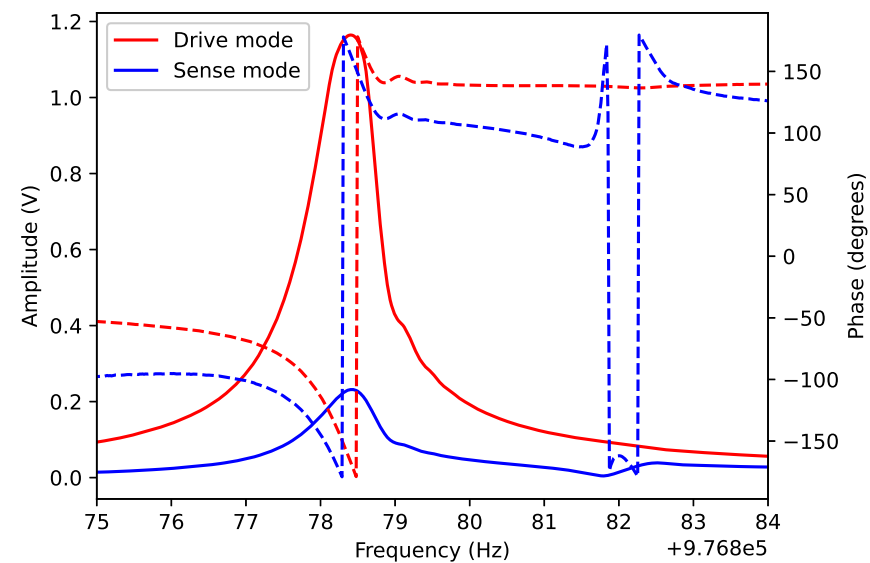

(a)

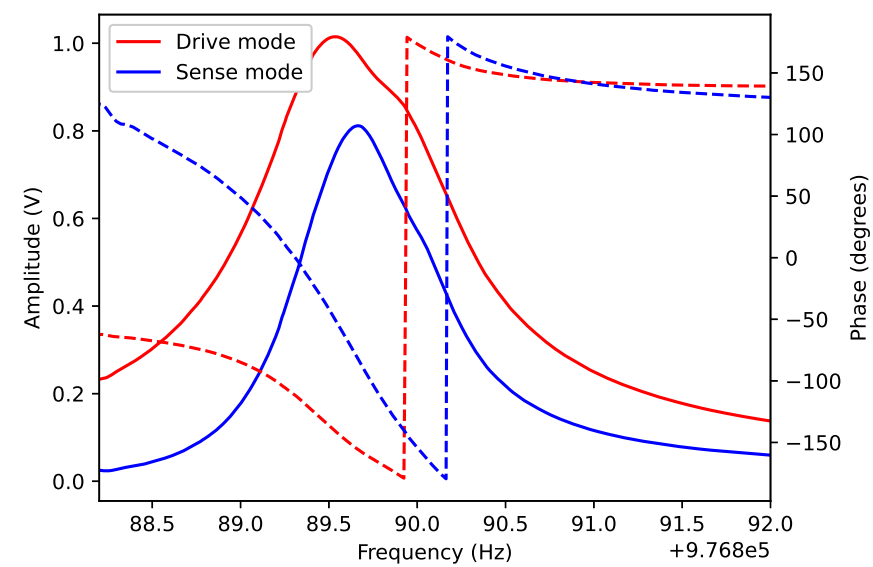

(b)

Fig. 7. Frequency response for the drive and sense modes of the device (a) before applying electrostatic tuning and (b) after applying a tuning voltage to reduce the frequency spacing between the modes. Amplitudes are represented with solid lines and phases are represented with dashed lines.

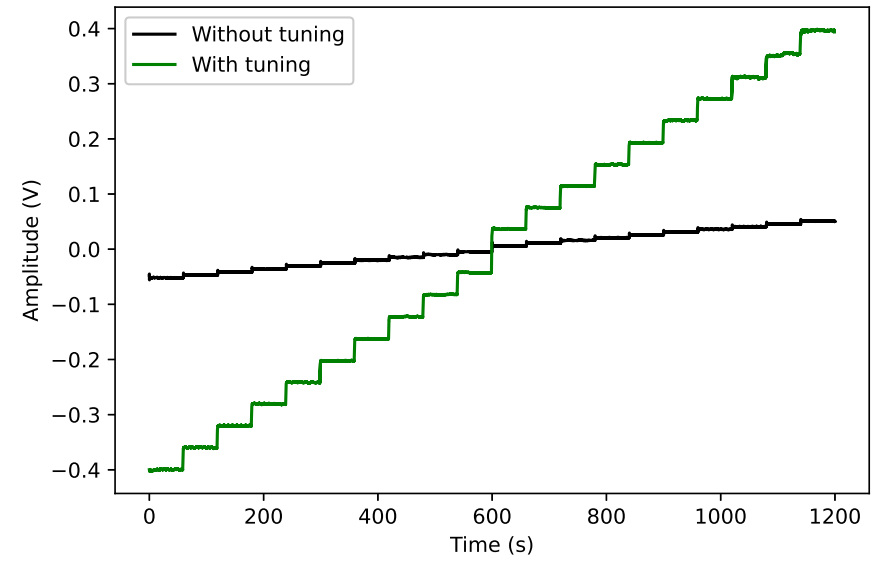

Fig. 8. Sense mode response from $-10 \mathrm{rpm}$ to $+10 \mathrm{rpm}$ with and without tuning applied to match the two modes.

set at $\pm 60 \% \mathrm{~s}, \pm 54^{\circ} / \mathrm{s}, \pm 48 \% \mathrm{~s}, \pm 42 \% \mathrm{~s}, \pm 36^{\circ} \% \mathrm{~s}, \pm 30^{\circ} / \mathrm{s}$ $\pm 24 \% \mathrm{~s}, \pm 18 \% \mathrm{~s}, \pm 12 \% \mathrm{~s}, \pm 6 \% \mathrm{~s}$ for a 1 minute interval in each case.

The sense mode responses to the input rotation about the sensitive axis with and without tuning, are shown in Fig. 8. Fig. 8 shows a linear increment in the output amplitude for every $6 \%$ increment in angular inputs. The increment in output amplitude is twice as big at $600 \mathrm{~s}$ when the transition of angular input is from $-6 \% \mathrm{~s}$ to $+6 \%$ s.

The mean value is calculated at each input and it is plotted in Fig. 9. The linear fitting of all the mean values shows that the quadrature error is compensated by adjusting $\phi_{y}$ in demodulator 1 . The scale factor of the gyroscope without tuning the drive and the sense mode is $850 \mu \mathrm{V} /\left({ }^{\circ} / \mathrm{s}\right)$. When tuned, the scale factor shows an improvement of 7.76 times, reaching $6600 \mu \mathrm{V} /\left({ }^{\circ} / \mathrm{s}\right)$. Using equation 2 , the predicted improvement in mechanical sensitivity when $\Delta f$ is reduced from $4.06 \mathrm{~Hz}$ to $0.4 \mathrm{~Hz}$ is approximately 7.9 times. Thus, the scale factor improvement achieved experimentally is in line with the

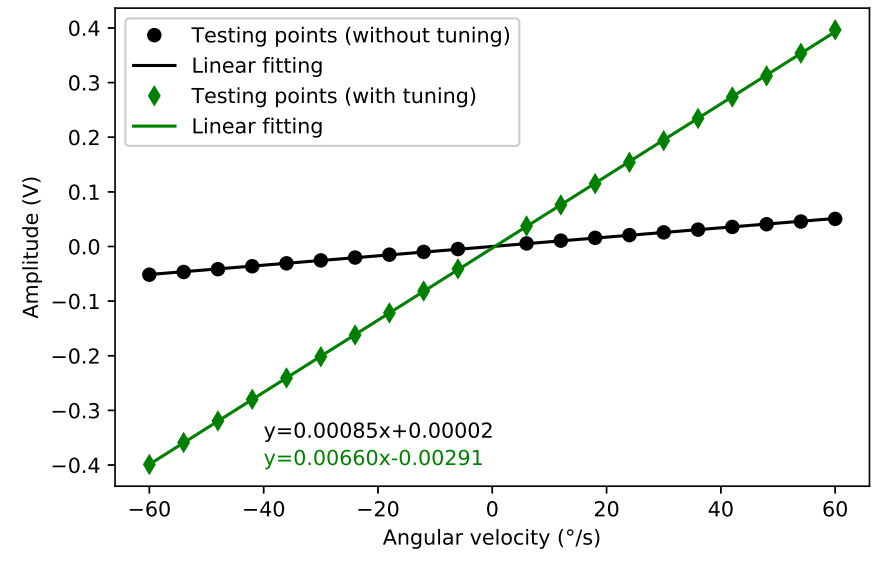

Fig. 9. Output response of the gyroscope to rotation rate ranging from $-60 \%$ to $60 \%$ s.

analytical prediction.

The device shows quality factor of $1.56 \mathrm{M}$ at $976 \mathrm{kHz}$ providing for an open-loop bandwidth of $0.31 \mathrm{~Hz}$. The dynamic range of the device for mode-mismatched and mode-matched operation are around $\pm 180 \%$ and $\pm 90 \%$ r respectively. Note that rotation rates higher than $180 \%$ were not tested in this work.

Fig. 10a shows the Allan deviation plot of the edgeanchored BAW gyroscope. The sensor exhibits an angle random walk (ARW) of $0.042 \% \sqrt{h}$ and a bias instability (BI) of $1.65 \%$. The ARW and BI are improved to $0.037 \% \sqrt{h}$ and $0.95 \% / \mathrm{h}$ respectively when the device is operated in a near mode-matched configuration as shown in Fig. 10b. The peak in the Allan deviation plot at mode-matched operation (Fig. $10 \mathrm{~b}$ ) is because of low frequency noise of the power source.

The ARW and BI are not proportionately improved with the increased mechanical sensitivity upon mode-matching for several possible reasons. Firstly, the device is tuned using an open-loop approach. The lack of a closed-loop implementation 


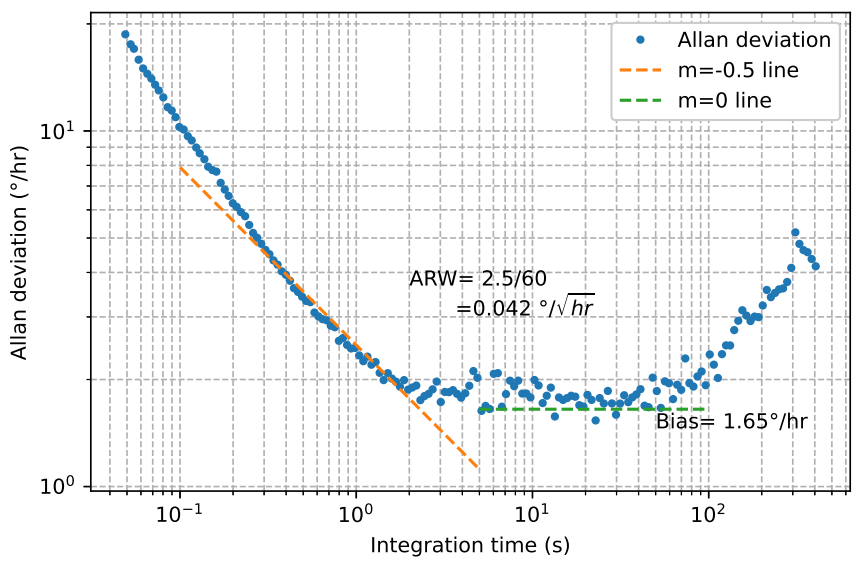

(a)

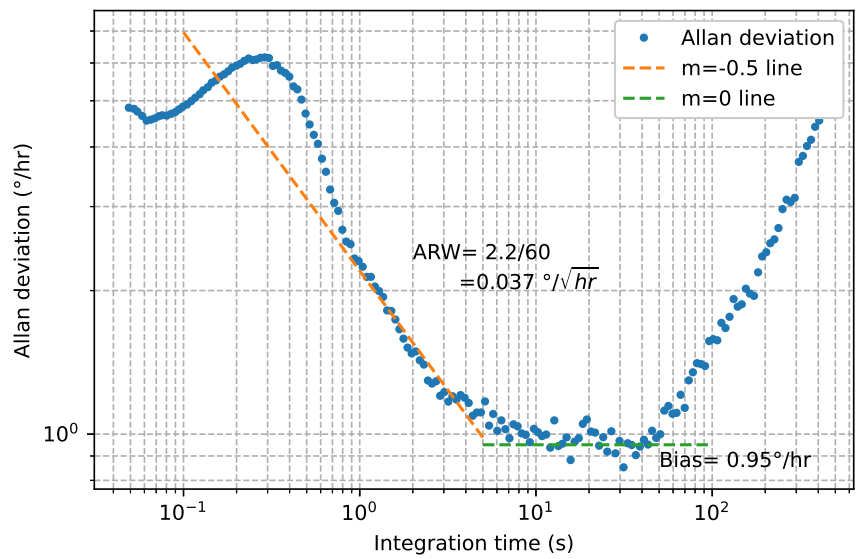

(b)

Fig. 10. Allan deviation plot for the MEMS gyroscope (a) without mode matching (b) with mode matching.

including automatic mode-matching can result in scale factor instability and increase in drive instabilities coupling into the sense channel. Secondly, the external tuning voltage source employed for resonance frequency tuning is noisy and this is operative only during mode-matching.

Equation 5 estimates the Brownian noise equivalent ARW for a MEMS Coriolis vibratory gyroscope [2].

$$
\mathrm{ARW}_{\text {Brown }}=\frac{1}{2 A_{\mathrm{g}} x_{0}} \sqrt{\frac{k_{B} T}{2 \pi m_{\text {eff }} f_{0} Q}} \times\left(\frac{180}{\pi} \times 60^{\circ} / \sqrt{h}\right)
$$

The angular gain $A_{g}$ is the ratio of the change in the vibration pattern angle to the applied angle of rotation $\left(\Omega_{z}\right)$ and depends on the sensor structure and nature of the resonant modes in operation. The $A_{g}$ for the secondary elliptic modes of a solid disk structure can be estimated as 0.23 [30]. The estimated displacement $x_{0}$ for the drive parameters employed (bias voltage of $90 \mathrm{~V}$ and ac drive voltage of $0.3 \mathrm{~V}$ ) is 0.18 $\mu \mathrm{m}$ and the effective mass $m_{\text {eff }}$ is estimated to be $0.48 \mathrm{mg}$. Using the measured $\mathrm{Q}$ and resonant frequency, the theoretical Brownian noise of this device is estimated to be approximately $0.0015 \% \sqrt{h}$ using equation 5. The measured ARW and BI of $0.037 \% \sqrt{h}$ and $0.95 \% / h$ respectively show that the current results are not dominated by thermo-mechanical noise. Further work is addressing noise and drift of the discrete readout and drive electronics and the noise and stability of the bias and tuning voltage sources employed.

\section{CONCLUSION}

This paper presents experimental results demonstrating improved sensitivity relative to the untuned case of a BAW disk gyroscope by implementing mode matching using electrostatic frequency tuning. By operating the gyroscope in a modematched operation, its sensitivity is improved by 7.76 times. The ARW of $0.037 \% \sqrt{h}$ and BI of $0.95 \% / h$ for a prototype device show that with improved front-end electronics and few iterations of the design, it is possible to develop highend MEMS gyroscopes based on this approach. Table III shows that the result of this work compares favorably with the literature on current bulk acoustic mode disk gyroscopes.

TABLE III

COMPARISON OF ARW AND BI FOR BULK MODE MEMS GYROSCOPES

\begin{tabular}{|c|c|c|}
\hline Reference & ARW $\left({ }^{\circ} / \sqrt{\mathbf{h}}\right)$ & BI $\left(^{\circ} / \mathbf{h}\right)$ \\
\hline Wen et al. $[3]$ & 0.06 & 0.85 \\
\hline Ayazi et al. [7] & 0.06 & 0.85 \\
\hline Serrano et al. $[13]$ & 0.39 & 3.5 \\
\hline This work & $\mathbf{0 . 0 3 7}$ & $\mathbf{0 . 9 5}$ \\
\hline
\end{tabular}

Future work will improve the noise floor by optimizing the drive and readout electronics and using low noise voltage sources for both bias and tuning voltages. In addition, BI can be further improved by optimizing the packaging for immunity to environmental drift [31] and by implementing improved temperature compensation and control schemes. The ARW and BI under mode-matched conditions can be improved by implementing in-run automatic mode matching [32]. By optimizing these factors a noise floor nearer the thermomechanical noise limit can be implemented as a step towards navigation grade MEMS gyroscopes.

\section{ACKNOWLEDGMENT}

Authors would like to thank Jiangkun Sun for helpful discussions. This work was supported by funding from Engineering and Physical Research Council UK, Silicon Microgravity and Innovate UK.

\section{REFERENCES}

[1] A. D. Challoner, H. H. Ge, and J. Y. Liu. Boeing disc resonator gyroscope. In 2014 IEEE/ION Position, Location and Navigation Symposium - PLANS 2014, pages 504-514, 2014.

[2] L. Qingsong, X. Dingbang, Z. Xin, X. Yi, Z. Ming, H. Zhanqiang, H. Kaixuan, Z. Yongmeng, and W. Xuezhong. 0.04 degree-per-hour mems disk resonator gyroscope with high-quality factor $(510 \mathrm{k})$ and long decaying time constant (74.9 s). Microsystems \& nanoengineering, 4(1):1-11, 2018. 
[3] H. Wen, A. Daruwalla, C. Liu, and F. Ayazi. A hermetically-sealed $2.9 \mathrm{mhz} \mathrm{N}=3$ disk baw gyroscope with sub-degree-per-hour bias instability. In 2020 IEEE 33rd International Conference on Micro Electro Mechanical Systems (MEMS), pages 741-744, 2020.

[4] P. Taheri-Tehrani, M. Kline, I. Izyumin, B. Eminoglu, Y. Yeh, Y. Yang, Y. Chen, I. Flader, E. J. Ng, T. W. Kenny, B. E. Boser, and D. A. Horsley. Epitaxially-encapsulated quad mass gyroscope with nonlinearity compensation. In 2016 IEEE 29th International Conference on Micro Electro Mechanical Systems (MEMS), pages 966-969, 2016.

[5] J. Cho, J. . Woo, J. Yan, R. L. Peterson, and K. Najafi. A high-q birdbath resonator gyroscope (brg). In 2013 Transducers Eurosensors XXVII: The 17th International Conference on Solid-State Sensors, Actuators and Microsystems (TRANSDUCERS EUROSENSORS XXVII), pages 18471850,2013

[6] T. Nagourney, J. Y. Cho, B. Shiari, A. Darvishian, and K. Najafi. 259 second ring-down time and 4.45 million quality factor in $5.5 \mathrm{khz}$ fused silica birdbath shell resonator. In 2017 19th International Conference on Solid-State Sensors, Actuators and Microsystems (TRANSDUCERS), pages 790-793, 2017.

[7] F. Ayazi, H. Wen, A. Daruwalla, and P. Gupta. Environmentallyrobust high-performance silicon timu chip. In 2020 IEEE/ION Position, Location and Navigation Symposium (PLANS), pages 16-23, 2020.

[8] X. Zou, C. Zhao, and A. A. Seshia. Edge-anchored mode-matched micromachined gyroscopic disk resonator. In 2017 19th International Conference on Solid-State Sensors, Actuators and Microsystems (TRANSDUCERS), pages 2183-2186, 2017.

[9] J. E. Y Lee, , , and A. A. Seshia. 5.4-mhz single-crystal silicon wine glass mode disk resonator with quality factor of 2 million. Sensors and Actuators A: Physical, 156(1):28-35, 2009.

[10] G. Sobreviela, X. Zou, C. Zhao, M. Pandit, and A. A. Seshia. An ultra-high-quality factor silicon disk resonator. In 2019 20th International Conference on Solid-State Sensors, Actuators and Microsystems Eurosensors XXXIII (TRANSDUCERS EUROSENSORS XXXIII), pages 527-530, 2019.

[11] M. Parajuli, G. Sobreviela, H. Zhang, and A. A. Seshia. Enhancement of frequency stability in injection locked bulk mode mems oscillators. In 2021 IEEE 34th International Conference on Micro Electro Mechanical Systems (MEMS), pages 941-944, 2021.

[12] M. Parajuli, G. Sobreviela, H. Zhang, and A. A. Seshia. A silicon mems disk resonator oscillator demonstrating 36 ppt frequency stability. In 202121 st International Conference on Solid-State Sensors, Actuators and Microsystems (Transducers), pages 305-308, 2021.

[13] D. E. Serrano, M. F. Zaman, A. Rahafrooz, P. Hrudey, R. Lipka, D. Younkin, S. Nagpal, I. Jafri, and F. Ayazi. Substrate-decoupled, bulkacoustic wave gyroscopes: Design and evaluation of next-generation environmentally robust devices. Microsystems \& Nanoengineering, 2(1):1-10, 2016.

[14] X. Wei and A. A. Seshia. Analytical formulation of modal frequency split in the elliptical mode of scs micromechanical disk resonators. Journal of Micromechanics and Microengineering, 24(2):025011, 2014.

[15] M. Parajuli, G. Sobreviela, and A. A. Seshia. Electrostatic frequency tuning of bulk acoustic wave disk gyroscopes. In 2020 Joint Conference of the IEEE International Frequency Control Symposium and International Symposium on Applications of Ferroelectrics (IFCS-ISAF), pages $1-4,2020$

[16] N. Yazdi, F. Ayazi, and K. Najafi. Micromachined inertial sensors. Proceedings of the IEEE, 86(8):1640-1659, 1998.

[17] C. Acar and A. Shkel. MEMS vibratory gyroscopes: structural approaches to improve robustness. Springer Science \& Business Media, 2008.

[18] A. S. Phani, A. A. Seshia, M. Palaniapan, R. T. Howe, and J. Yasaitis. Modal coupling in micromechanical vibratory rate gyroscopes. IEEE Sensors Journal, 6(5):1144-1152, 2006.

[19] I. P. Prikhodko, J. A. Gregory, W. A. Clark, J. A. Geen, M. W. Judy, C. H. Ahn, and T. W. Kenny. Mode-matched mems coriolis vibratory gyroscopes: Myth or reality? In 2016 IEEE/ION Position, Location and Navigation Symposium (PLANS), pages 1-4, 2016.

[20] C. Acar, A. R. Schofield, A. A. Trusov, L. E. Costlow, and A. M. Shkel. Environmentally robust mems vibratory gyroscopes for automotive applications. IEEE Sensors Journal, 9(12):1895-1906, 2009.

[21] H. Xie and G. K. Fedder. Integrated microelectromechanical gyroscopes. Journal of aerospace engineering, 16(2):65-75, 2003.

[22] A. Mustafazade, M. Pandit, C. Zhao, G. Sobreviela, Z. Du, P. Steinmann, X. Zou, R. T. Howe, and A. A. Seshia. A vibrating beam mems accelerometer for gravity and seismic measurements. Scientific reports, 10(1):1-8, 2020.
[23] Z. Hao, S. Pourkamali, and F. Ayazi. Vhf single-crystal silicon elliptic bulk-mode capacitive disk resonators-part i: design and modeling. Journal of Microelectromechanical Systems, 13(6):1043-1053, 2004.

[24] Y. Sun and M. Saka. Thermoelastic damping in micro-scale circular plate resonators. Journal of Sound and Vibration, 329(3):328-337, 2010.

[25] Z. Hao and F. Ayazi. Support loss in the radial bulk-mode vibrations of center-supported micromechanical disk resonators. Sensors and Actuators A: Physical, 134(2):582-593, 2007.

[26] H. Sumali. Squeeze-film damping in the free molecular regime: model validation and measurement on a mems. Journal of Micromechanics and Microengineering, 17(11):2231, 2007.

[27] F. Ayazi, L. Sorenson, and R. Tabrizian. Energy dissipation in micromechanical resonators. In Micro-and Nanotechnology Sensors, Systems, and Applications III, volume 8031, page 803119. International Society for Optics and Photonics, 2011.

[28] S. D. Bindel and S. Govindjee. Elastic pmls for resonator anchor loss simulation. International Journal for Numerical Methods in Engineering, 64(6):789-818, 2005.

[29] J. T. M. Van Beek and R. Puers. A review of mems oscillators for frequency reference and timing applications. Journal of Micromechanics and Microengineering, 22(1):013001, 2011.

[30] H. Johari. Micromachined Capacitive Silicon Bulk Acoustic Wave Gyroscopes. PhD thesis, Georgia Institute of Technology, 2008.

[31] M. Pandit, A. Mustafazade, G. Sobreviela, C. Zhao, X. Zou, and A. A. Seshia. Experimental observation of temperature and pressure induced frequency fluctuations in silicon mems resonators. Journal of Microelectromechanical Systems, pages 1-6, 2021.

[32] C. Li, H. Wen, S. Wisher, A. Norouzpour-Shirazi, J.Y. Lei, H. Chen, and F. Ayazi. An fpga-based interface system for high-frequency bulkacoustic-wave microgyroscopes with in-run automatic mode-matching. IEEE Transactions on Instrumentation and Measurement, 69(4):1783$1793,2019$.

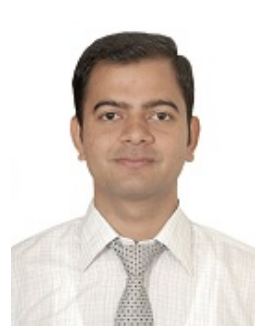

Madan Parajuli received the B.E. in Electrical and Electronics Engineering from Anna University, India, in 2015. He obtained a MSc degree in Joint International Master in Smart Systems Integration, jointly awarded by Heriot-Watt University in Edinburgh, Budapest University of Technology and Economics, and University of South-Eastern Norway in 2018. He is pursuing a Ph.D. degree with the MEMS Research Group, Nanoscience Centre, Department of Engineering, University of Cambridge, U.K.. His current research focuses on high performance microelectromechanical systems (MEMS) gyroscopes, and his research interests include MEMS inertial sensors and oscillators.

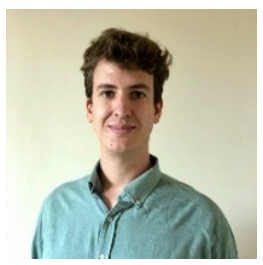

Guillermo Sobreviela was born in Huesca, Spain, in 1989. He received his BSc in Physics from the University of Zaragoza, Zaragoza, Spain, in 2012, and his MSc in Micro and Nanoelectronics Engineering in 2013. He was awarded his $\mathrm{PhD}$ in Electronics from the Universitat Autònoma de Barcelona, Barcelona, Spain, in 2016. From 2016 to 2019 he worked as a full-time Research Associate in microsystems (MEMS) at the University of Cambridge, UK, where he was involved in the research and development of MEMS inertial sensors. He is currently working as a MEMS Design Engineer at Silicon Microgravity LTD, Cambridge, UK, where he is developing ultra-high-resolution MEMS gravimeters. His current research interests include MEMS micro-resonators for resonance tracking-based MEMS sensors (such as inertial sensors) and actuators. This research encompasses the design and manufacture of the MEMS device as well as the system implementation of the sensor. 


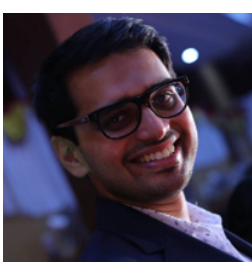

Milind Pandit (GS'16-M'18) received his BASc degree in electrical and electronics engineering with a specialization in Micro and Nano technology from the University of British Columbia, Canada. His Ph.D. degree was conducted on microelectromechanical systems (MEMS) and inertial sensors design with emphasize on coupled resonators topology at the University of Cambridge, UK. He is currently working as a MEMS Design and Analytics engineer at Silicon Microgravity Ltd. where he is optimizing the resolution of high-performance inertial sensors by focusing on packaging, environmental control, and interface electronics.

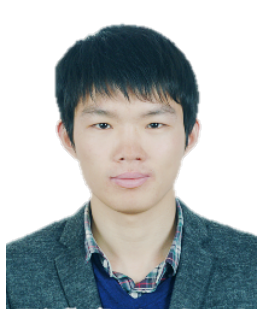

Hemin Zhang received the B.E. degree in electrical engineering and the Ph.D. degree in microelectromechanical systems and nanotechnology from the Northwestern Polytechnical University, Xi'an, China, in 2011 and 2017, respectively. He was with the ESYCOM lab, ESIEE Paris and the Univ Gustave Eiffel, France, as a postdoc from Sept. 2017 to Mar. 2019. He joined the Nanoscience Center in the University of Cambridge, U.K., in Apr. 2019 as a research associate. He is now a Marie Curie Fellow at ESAT, KU Leuven. He was the recipient of the Outstanding Paper Award Finalist at the Transducers 2015, the Outstanding Paper Award Winner at the IEEE MEMS 2016. His current research interests include MEMS resonators, coupled resonators, mode-localized sensors, kinetic energy harvesters and their conditioning circuits.

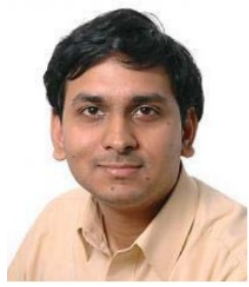

Ashwin A. Seshia (S'98-M'02-SM'10-F'21) received the B.Tech. degree in engineering physics from IIT Bombay, Mumbai, India, in 1996, the M.S. and $\mathrm{Ph} . \mathrm{D}$. degrees in electrical engineering and computer sciences from the University of California at Berkeley, Berkeley, CA, USA, in 1999 and 2002, respectively, and the M.A. degree from the University of Cambridge, Cambridge, U.K., in 2008. He was with the Berkeley Sensor and Actuator Center, University of California at Berkeley. In 2002, he joined the faculty of the Engineering Department with the University of Cambridge, where he is currently a Professor in Microsystems Technology and a Fellow of Queens' College.

His research interests include microengineered dynamical systems with applications to sensors and sensor systems. Dr. Seshia is a Fellow of the Institute of Physics and the Institution for Engineering and Technology. $\mathrm{He}$ serves on the editorial boards for the IEEE Journal of Microelectromechanical Systems, and IEEE Transactions on Ultrasonics, Ferroelectrics, and Frequency Control. 